\title{
Shot hole encourages almond drop, doesn't harm kernels
}

\author{
Beth L. Teviotdale \\ a Nancy Goodell \\ Dennis Harper
}

\begin{abstract}
Severe spring outbreaks of shot hole disease are often accompanied by leaf fall and fruit drop. Although fruit are shed in response to defoliation, the direct effect of shot hole infection on developing fruit was not known. When fruit at various stages of development were inoculated with high concentrations of the shot hole pathogen, very young and midsized fruit fell, whereas older fruit were retained. Typical shot hole lesions developed on midsized fruit, and full-sized fruit became resistant to infection when embryo development began. Infection did not affect kernel weight, but caused a slight, economically unimportant reduction in kernel length.
\end{abstract}

Shot hole disease of stone fruit trees, caused by the fungus Wilsonomyces carpophilus, produces lesions on leaves, fruit, flowers and succulent shoots. Leaf lesions that abscise leave behind small holes, or "shot holes," the symptom for which the disease is named. Leaf infection that leads to defoliation is the most serious aspect of shot hole disease on almond, Prunus dulcis, because severe defoliation during early fruit development can cause young fruit to fall, and repeated defoliation weakens trees and reduces yield. Extended rainfall in spring contributes to severe shot hole outbreaks, and growers regularly treat almond trees with fungicides to control the disease.

Fruit infections produce small, corky, slightly raised lesions on the hull, which sometimes extrude gum. Coalesced infections may deform the fruit, but the kernel is unharmed. Internal gumming, which can have several causes, produces dried gum de- posits on the kernel, imparting a lacquered appearance to the kernel and rendering it unmarketable. Casual observations suggested that such lacquered kernels might also be caused by shot hole infections.

Past research attributing yield loss to shot hole disease recorded higher yields and lower disease incidence from fungicide-treated than from untreated trees. Because kernel quality was similar in fruit from trees with differing disease incidence, yield loss was credited to presumed fruit abscission induced in spring by defoliation or by general weakness. These conclusions were based on measurements of fruit samples collected at harvest from trees with varying amounts of leaf and fruit infection, not on evaluation of disease effects on individual fruit. Furthermore, disease levels were determined on mature fruit in summer, and effects on younger fruit in spring were not measured.

Almond trees finish bloom in early March. Unpollinated and unfertilized fruit grow about another 3 weeks, to 5 to $10 \mathrm{~mm}$ long, and then fall from the tree. Fertilized fruit continue to lengthen but remain soft until they attain full size at the end of April or early May, when embryo development and shell hardening ensue. A portion of the fertilized fruit are shed in March and April during fruit elongation and before they reach maximum size. Until they begin to yellow and cease to lengthen, fruit destined to drop prematurely are indistinguishable from those that will be retained.

Fruit at different stages of development perhaps vary in susceptibility and response to infection. Infected young fruit may fall or produce kernels of lesser quality, and kernel quality may be influenced by the severity
Fruit growth stage when shot hole infection is likely to cause fruit drop.

of infection. Our objectives were to determine the effects of shot hole disease on fruit abscission and kernel quality of almond fruit inoculated with $W$. carpophilus at different stages of fruit development.

\section{Experimental orchard at Kearney}

We conducted the experiments in almond orchards planted with cultivars 'Mission' and 'Nonpareil' or 'Carmel' and 'Nonpareil', located at the UC Kearney Agricultural Center in Fresno County. Trees were mature, were flood furrow irrigated and were not treated with fungicides.

Experimental design and analysis. In each experiment, each of the four replications was located in one quadrant of a tree, and different trees were used on each inoculation date. Data from repeated experiments were combined for analysis. An arcsine transformation was performed on data for percent fruit abscission before analysis of variance. The means reported are for main effects or for data combined over years and were separated by orthogonal contrasts.

Fruit maturity when inoculated. We inoculated 'Mission' almond fruit once at approximately weekly intervals on March 26 and April 5, 12, 19 and 26, 1990, and on March 18 and 24, and April 8, 16 and 23, 1993. Inoculations began as fruit emerged from the floral cup and continued until they reached full size ( $34.8 \mathrm{~mm}$ average length) at the onset of embryo development. We used fruit length as an indication of fruit age. The average length of 20 fruit selected arbitrarily from among inoculated fruit, measured in millimeters with a ruler on each inoculation date, was $8.6,17.1$, 24.2, 31.3 and 34.1 in 1990 and 10.4, 18.0, 27.4, 31.5 and 35.6 in 1993. 
Sixty fruit per replication were thoroughly wetted with water using a Nalgene aerosol spray bottle and, using a similar sprayer, each fruit was inoculated immediately with 0.5 to 1.0 $\mathrm{ml}$ (greater volume was required to cover larger fruit) suspensions containing $10^{3}, 10^{4}$ or $10^{5}$ conidia per milliliter (cpm). Inoculated and uninoculated control fruit were kept wet for 48 hours after inoculation by a misting system that produced a fine spray. The misting system consisted of standard drip irrigation hose strung through the tree canopy and fitted with fan-jet $340^{\circ}$ misting nozzles that delivered 2.0 gallons of water per hour under $30.0 \mathrm{psi}$. The system was operated by an automatic timer that released water for 2 minutes at 10-minute intervals.

Fruit infection and abscission were assessed on April 24, 1990, and on April 13, 1993, in treatments that had been made by those dates. Each fruit was gently tapped, and those that fell were returned to the laboratory and inspected for shot hole infection. Tissue samples from 20 representative fruit were cultured, and W. carpophilus was isolated from $50 \%$ to $90 \%$ of the samples. The pathogen was recovered from some young fruit that had not developed typical shot hole lesions, and was more easily cultured from areas of coalesced than discrete lesions. Areas of coalesced lesions were most abundant $(40 \%$ to $60 \%)$, occasional (less than $10 \%$ ) and absent on fruit inoculated with $10^{5}, 10^{4}$ and $10^{3} \mathrm{cpm}$, respectively. No fruit were deformed.

We evaluated final fruit abscission in all treatments by counting the number of fruit that were firmly attached to the trees on May 24, 1990, and June 11,1993 . Disease severity on 10 (or all that were present if fewer than 10 remained) fruit gathered from each replication was determined on May 29, 1990, and June 15, 1993.

Fruit abscission was significantly $(P=0.001)$ less in $1990(40.5 \%)$ than in $1993(50.2 \%)$, and the average number of lesions per fruit (10.5 in 1990 and 9.2 in 1993) did not differ. As fruit age (as determined by length) when inoculated increased, the percentage of fruit abscission decreased $(P=0.001$, fig.
1A). Lesions were more abundant on fruit inoculated when midsize (average 25.8 to $31.4 \mathrm{~mm}$ ) than when smaller or larger $(P=0.004$, fig. $1 B)$. Increasing inoculum concentration was accompanied by an increase in fruit abscission and average number of lesions per fruit only when fruit of intermediate development were inoculated $(P=0.001)$. Differing inoculum concentrations did not cause significant differences in fruit abscission or lesion numbers on inoculated young or full-sized fruit. There were more $(P=0.001)$ dropped fruit and lesions in inoculated than in uninoculated treatments.

Kernel quality. The effect of shot hole infection on kernel quality of fruit inoculated at various stages of development was examined. Seventy 'Carmel' fruit in each of four replications were inoculated once with a suspension of $10^{5} \mathrm{cpm}$ on March 28 and April 4, 13 and 27, 1994, and on March 26 and April 6, 14 and 29, 1995. Fruit on a separate tree were inoculated three times, on the last three dates each year, to augment the severity of hull infection. Uninoculated control fruit were included on each inoculation date and with the repeated inoculation treatment. Trees were misted for 48 hours after inoculation, as described above. The average fruit length $(\mathrm{mm})$ when inoculated on each respective inoculation date was 25.4, 30.4, 33.4 and 38.3 in 1994 and 24.7, 29.0, 32.1 and 38.4 in 1995.

Disease severity on 10 fruit per replication was evaluated on June 23 , 1994, and July 6, 1995. There were more lesions per fruit in 1994 (27.1) than in 1995 (7.7), and more lesions on inoculated (34.4) than on uninoculated $(0.5)$ fruit $(P=0.0001)$. The average number of lesions per fruit decreased as average fruit length at inoculation increased, and more lesions were produced by multiple than by single inoculations (fig. 2A).

All remaining fruit were harvested on Aug. 29, 1994, and Aug. 26, 1995. We removed the kernels of 50 fruit per replication and assessed the percentage that had gum deposits (lacquered) or were incompletely filled (shriveled).
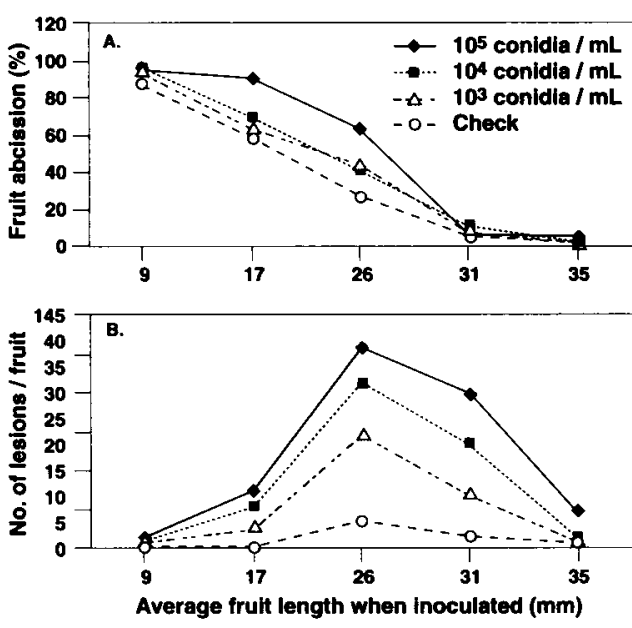

Fig. 1. Effect of inoculum concentration (conidia $/ \mathrm{ml}$ ) on percentage of fruit abscission (A) and number of lesions per fruit (B) of 'Mission' almond fruit inoculated with the shot hole pathogen, Wilsonomyces carpophilus. Data from 1990 and 1993 combined.
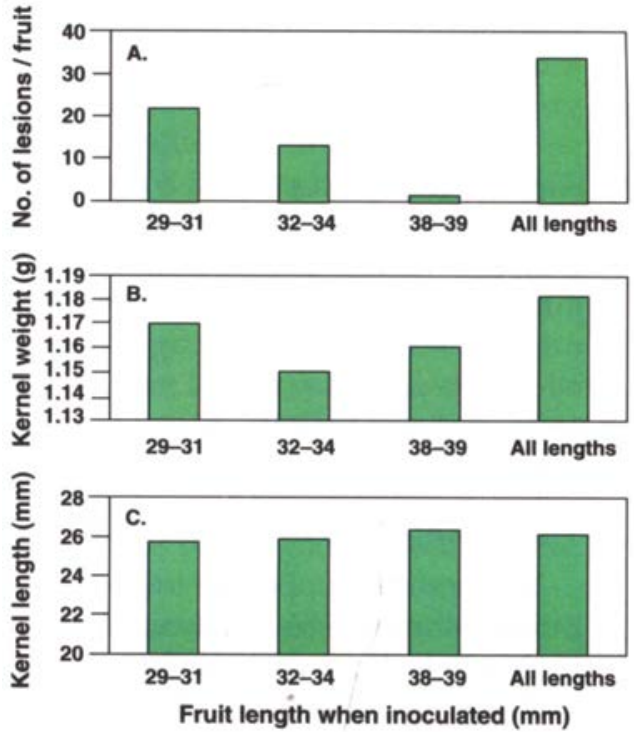

Fig. 2. Effect of fruit maturity (length) when inoculated on kernel quality of 'Carmel' almond fruit inoculated with the shot hole pathogen, Wilsonomyces carpophilus. Fruit inoculated once when 29 to 31,32 to 34 , or 38 to $39 \mathrm{~mm}$ long or three times (all lengths). Average number of lesions per fruit (A), kernel weight (B) and kernel length (C). Data from 1994 and 1995 combined.

Shriveled or lacquered kernels did not exceed $2.5 \%$ in any treatment in 1994 and were not observed in 1995. Lacquered and shriveled kernels were replaced with healthy kernels, so that 50 healthy kernels were air dried for 72 hours at $149^{\circ} \mathrm{F}\left(65^{\circ} \mathrm{C}\right)$ in a forced air oven and then weighed and measured.

Average kernel weight and length were greater in $1994(1.21 \mathrm{~g}$ and 26.5 $\mathrm{mm}$ ) than in 1995 (1.12 $\mathrm{g}$ and $25.2 \mathrm{~mm})$ 
$(P=0.001)$. Average kernel weight of inoculated $(1.11 \mathrm{~g})$ and uninoculated $(1.12 \mathrm{~g})$ fruit did not differ significantly, but kernel length was shorter in inoculated $(25.6 \mathrm{~mm})$ than in uninoculated $(26.1 \mathrm{~mm})$ fruit $(P=0.001)$. The length of fruit when inoculated did not influence the weight (fig. 2B) or length (fig. 2C) of ripe kernels at harvest.

Abscission among fruit inoculated on the first date each year was $76.7 \%$ in 1994 and $84.3 \%$ in 1995, and among uninoculated controls was $52.6 \%$ in 1994 and $68.7 \%$ in 1995 . Too few inoculated fruit in these categories remained to assess fruit quality. Fruit drop was less than $6.0 \%$ among treatments following the first one.

Weather data were collected from a California Irrigation Management Information System station located approximately $1 / 2$ mile from the experimental orchards at the Kearney Agricultural Center. Rainfall during bloom (late February through early March) was $17.3 \mathrm{~mm}$ in 1990, 53.3 $\mathrm{mm}$ in 1993, $9.1 \mathrm{~mm}$ in 1994 and 31.2 $\mathrm{mm}$ in 1995. Rainfall during the inoculation period was $24.1 \mathrm{~mm}$ in $1990,76.4 \mathrm{~mm}$ in $1993,40.4 \mathrm{~mm}$ in 1994 and $20.3 \mathrm{~mm}$ in 1995.

\section{Three stages of susceptibility}

Almond fruit appeared to go through three stages of susceptibility to shot hole. Very young infected fruit were apt to fall, and hull lesions were scarce on remaining fruit. Infected midsize fruit also fell, but survivors exhibited typical hull lesions. When fruit approached full size at the onset of embryo development, they were least susceptible. At this stage, abscission ceased and few hull lesions formed.

It is difficult to assess with confidence the response of very young fruit to infection because the majority of inoculated and uninoculated fruit that were present shortly after bloom eventually abscised. This fruit loss was probably caused in part by natural thinning and poor pollination. Almond trees are not self-fertile, so pollen carried by bees from the companion 'Nonpareil' trees was required to set fruit on the 'Mission' trees. Peak bloom of 'Nonpareil' trees generally occurs earlier than that of 'Mission' trees. Therefore these cultivars are not ideal pollenizers for eàch other. This, coupled with the greater amount of rain that fell during bloom in 1993 , which may have interrupted bee flight and pollination, could account for the higher abscission rate among small fruit observed that year than in 1990 . Also, the 48 hours of continuous wetness during the misting treatments may have contributed to the excessive abscission of very young fruit. We were not able to include a dry nonmisted control for comparison on each tree because the entire tree was wetted when misted. As fruit aged, selection of viable fruit was easier and percent abscission among the test fruit lessened. In the kernel-quality experiments on 'Carmel' trees, the higher average number of lesions per fruit found in 1994 perhaps reflected the greater amount of rain that fell during the inoculation period that year than in 1995.

Typical discrete shot hole lesions were uncommon among the dropped young fruit collected in April. Instead, areas of coalesced lesions or no obvious shot hole symptoms were found, and the pathogen was usually recovered from these. Most characteristic shot hole lesions on almond fruit resulted from infection of fruit intermediate in development. The means by which shot hole infection caused fruit drop was not obvious, because visible infections were not aggregated near the attachment to the pedicel. Shot hole lesions developed on leaves close to our inoculated fruit, but all shoots and spurs remained fully foliated and no obvious leaf loss was observed in any of our treatments.

Fruit abscission is clearly a component of yield loss. As mentioned earlier, defoliation can trigger loss of healthy and diseased fruit. This response, along with reduction of tree vigor and fruiting wood that follow repeated defoliation, has been viewed as the basis for yield loss from shot hole disease. Our experiments show that abscission of infected young fruit may also be independent of defoliation. In addition to yield reduction sustained as a consequence of defoliation, fruit may be lost directly to infection without substantial defoliation:
The effect of shot hole infection on kernel quality was negligible. Even multiple infections and coalesced lesions, more severe than often encountered in commercial orchards, did not reduce kernel weight or cause excessive shriveling or lacquering of the kernels of 'Carmel'. The slight (0.5 $\mathrm{mm}$ ) reduction in average kernel length found among inoculated fruit is not of economic importance, and we do not consider it a lessening of kernel quality. It does, however, suggest that kernel quality may be adversely affected by shot hole infections under some circumstances.

Fruit and leaves of almond trees are most likely to abscise if infected when young. When fruit are immature, many leaves on the tree are also young. In California, this stage of development coincides with the prolonged spring rains that favor shot hole. Infection by W. carpophilus at this time can result in defoliation and heavy fruit loss. Therefore it is important to protect almond trees from shot hole infection during this susceptible period.

B.L. Teviotdale is Extension Plant $\mathrm{Pa}$ thologist, N. Goodell is Staff Research Associate and D. Harper is former Staff Research Associate, UC Davis, stationed at Kearney Agricultural Center, Parlier.

The authors thank Valeshia Hines, Don Self, Andrew Nunez, Tom Turini and Andrea Chiamori for technical assistance and the Almond Board of California for support for this research.

\section{Further reading}

Highberg LM, Ogawa JM. 1986. Yield reduction in almond related to incidence of shot hole disease. Plant Dis 70:825-8.

Kester DE. 1996. Growth and development. In: Almond Production Manual. Division of Agriculture and Natural Resources, Univ of Calif Publ 3364. p 90-7.

Shaw DA, Adaskaveg JE, Ogawa JM. 1990. Influence of wetness period and temperature on infection and development of shot-hole disease of almond caused by Wilsonomyces carpophilus, Phytopathology 80:749-56.

Teviotdale BL, Goodell N, Harper D. 1997. Abscission and kernel quality of almond fruit inoculated with the shot hole pathogen Wilsonomyces carpophilus. Plant Dis 81:1454-8.

Teviotdale BL, Viveros M, Freeman MW, Sibbett GS. 1989. Effect of fungicides on shot hole disease of almonds. Cal Ag 43:21-3. 